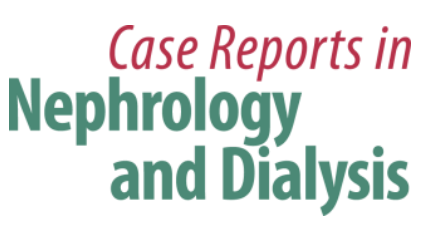

Case Rep Nephrol Dial 2018;8:239-245

DOI: 10.1159/000493188

Published online: November 14, 2018

(C) 2018 The Author(s)

Published by S. Karger AG, Base

www.karger.com/cnd

This article is licensed under the Creative Commons Attribution-NonCommercial 4.0 International License (CC BY-NC) (http://www.karger.com/Services/OpenAccessLicense). Usage and distribution for commercial purposes requires written permission.

\title{
Peritoneal Dialysis Exit Site Pyoderma Gangrenosum: A Case Report
}

\author{
Salman Anwar ${ }^{a} \quad$ Karen Holfeld $^{b} \quad$ Bhanu Prasad $^{c}$ \\ ${ }^{a}$ College of Medicine, University of Saskatchewan, Regina Campus, Regina, SK, Canada; \\ bSection of Dermatology, Department of Medicine, Regina, SK, Canada; 'Section of \\ Nephrology, Department of Medicine, Regina, SK, Canada
}

\section{Keywords}

Peritoneal dialysis · Pyoderma gangrenosum - End-stage renal disease · Inflammatory dermatosis

\begin{abstract}
Background: Pyoderma gangrenosum (PG) is a rare, chronic inflammatory condition exhibiting mucopurulent or hemorrhagic exudates. The majority of cases are associated with inflammatory bowel disease, rheumatological diseases, and hematological malignancies. In the absence of typical serological markers and specific histopathological changes, the diagnosis is often clinical. Being rare, it is frequently misdiagnosed, which leads to a delay in instituting appropriate therapy. Case Presentation: We present a 53-year-old male of Aboriginal descent with end-stage renal disease due to diabetes who underwent insertion of a peritoneal dialysis (PD) catheter. Five weeks after PD catheter insertion, he started to notice a painful ulcer surrounded by a bed of erythema. The lesion eventually progressed to a purulent, hemorrhagic ulcer surrounded by a raised, irregular, violaceous border along the entirety of the PD catheter tunnel. There was no history of underlying systemic diseases commonly associated with PG. The catheter was removed, and an elliptical biopsy was taken, which ruled out infection, malignancy, and vasculitis. The changes were felt to be consistent with PG. The patient underwent treatment initially with topical corticosteroids, followed by oral prednisone, which unfortunately worsened his diabetic control; due to this, he was transitioned to cyclosporine, with complete resolution. Conclusions: Lesions at the PD catheter exit site are usually treated for
\end{abstract}




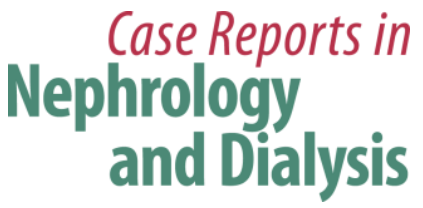

Case Rep Nephrol Dial 2018;8:239-245

DOI: $10.1159 / 000493188$

(c) 2018 The Author(s). Published by S. Karger AG, Basel www.karger.com/cnd

Anwar et al.: Peritoneal Dialysis Exit Site Pyoderma Gangrenosum: A Case Report

infections. However, for ulcers that are painful, rapidly expanding, nonhealing, and unresponsive to antibiotics, PG should be considered as a differential diagnosis. This is the first reported case of PG occurring at the exit site of a PD catheter.

(C) 2018 The Author(s)

Published by S. Karger AG, Basel

\section{Background}

Pyoderma gangrenosum (PG) is a rare, inflammatory dermatosis that is often misdiagnosed [1]. While its etiology remains unknown, PG has been classified into four clinical variants, of which the most common is the classic ulcerative form [2]. The lesion typically begins as a painful erythematous nodule or acneiform pustule which progresses rapidly to form a necrotic ulcer surrounded by a raised, violaceous, irregular border [3]. Fifty to $70 \%$ of cases are associated with inflammatory bowel disease, rheumatological diseases, or hematological malignancies [4], and $25 \%$ of cases occur following incidental or iatrogenic trauma [5].

We report a case of ulcerative PG which developed at the exit site of a peritoneal dialysis (PD) catheter in an end-stage renal disease (ESRD) patient without any history of underlying systemic diseases commonly associated with PG. To our knowledge, a similar occurrence has not been documented in the literature thus far.

\section{Case Report}

A 53-year-old male of Aboriginal descent with a history of hypertension, coronary artery disease, and diabetic nephropathy leading to ESRD requiring chronic PD was seen in July 2016 for insertion of a double-cuffed PD catheter. The patient tolerated the procedure well without any immediate complications. Five weeks later, he noticed an ulcerated area $5 \mathrm{~mm}$ from the catheter exit site surrounded by a bed of mild erythema and swelling. The lesion improved marginally following oral cephalexin and topical mupirocin cream application to the affected area, but it eventually worsened (Fig. 1).

Given the lack of systemic signs of illness and occasional improvement of the lesion with antibiotics, it was treated as an ongoing exit site infection. The lesion gradually extended along the subcutaneous tunnel, with multiple small areas of ulceration on a bed of worsening purplish erythema. There was associated nonpurulent drainage from the ulcerating dermis (Fig. 2). As the ulcerated lesion failed to improve after 56 weeks of intermittent courses of antibiotics (Fig. 3), we decided to remove the catheter and offer renal replacement therapy in the form of hemodialysis.

Blood cultures taken at the time of PD catheter removal failed to reveal any growth after 5 days of incubation. A biopsy of the skin and subcutaneous tissue was sent for Gram staining, culture, and histological examination. The Gram stain of the biopsied ulcerated lesion was unremarkable, and the culture revealed a mixed flora. The histology report commented on focally ulcerated areas in the epidermis and on a sinus tract lined by inflamed granulation tissue extending into the deep dermis. There was no evidence of malignancy. The patient was reviewed by a dermatologist, who wondered whether the painful, indurated, and erythematous ulcerated sclerotic linear plaque on his lower abdomen was consistent with PG. The case was discussed with the histopathologist, and the microabscesses, fibrosis, and inflammation were felt to be due to PG.

Following the diagnosis of PG, topical and oral steroids were initiated, which led to an impressive resolution of the erythema and gradual healing of the ulcerated lesions (Fig. 4, 5). 


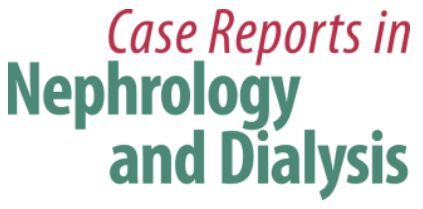

Case Rep Nephrol Dial 2018;8:239-245

DOI: $10.1159 / 000493188$

(c) 2018 The Author(s). Published by S. Karger AG, Basel www.karger.com/cnd

Anwar et al.: Peritoneal Dialysis Exit Site Pyoderma Gangrenosum: A Case Report

Unfortunately, the systemic steroids elevated the patient's blood glucose levels and made his diabetes difficult to manage. It was also noted that tapering the dose of systemic steroids led to worsening of the lesions, and the patient was transitioned to cyclosporine at approximately $5 \mathrm{mg} / \mathrm{kg}$ for a total dose of $150 \mathrm{mg}$ b.i.d. The introduction of a calcineurin inhibitor led to a sustained response and eventual resolution (Fig. 6).

\section{Discussion}

PG is a rare inflammatory condition exhibiting mucopurulent or hemorrhagic exudates. The lesion often begins as an ulcer exhibiting characteristics of a follicular pustule with surrounding erythematous, edematous, and painful skin which ultimately progresses to tissue necrosis [6]. The majority of cases (50-70\%) are associated with systemic illnesses including inflammatory bowel disease, hematological malignancies, hepatitis, seronegative rheumatoid arthritis, spondylitis, and monoclonal gammopathies [2]. Despite recent advances in the pathophysiology of the disease, the diagnosis is mainly clinical and by exclusion [7]. In our patient, we were unable to elicit any history of inflammatory bowel disease, the serological results for rheumatological diseases were negative, and there was no evidence of monoclonal gammopathy on serum protein electrophoresis.

Brunsting et al. [8] coined the term "pyoderma gangrenosum" in 1930 and hypothesized about a possible infectious etiology. Nine decades later, although its etiology remains uncertain, there are supporting data implying a dysregulation of the immune system $[9,10]$. In the absence of specific histopathologic features and serologic markers, the diagnosis of PG remains clinical. PG lesions mimic infection, vascular insufficiency ulcers, systemic vasculitis, cancer, and exogenous trauma [11]. A biopsy should be performed to rule out other causes of ulceration. There are no specific histological features of PG, and they vary based on the evolutionary stages of the disease.

While PG lesions are typically triggered by pathergy (incidental or iatrogenic trauma), this is the first reported association with the exit site of a PD catheter. Initially, we had felt that the patient had an infection of the PD catheter exit site. Our views were further strengthened by a perceptible response regarding the pain and erythema following the introduction of oral antibiotics. We continued to treat the patient as having an infection and wondered whether he was being diligent with aseptic techniques and exit site management. Our inability to recognize PG in this individual led to a delay in instituting appropriate therapy and led to gradual progression of the ulcerated areas, erythema, and intense pain.

The diagnosis of PG should be considered for all patients whose wounds are painful, rapidly expanding, nonhealing, and unresponsive to antibiotics, or worsening with surgical debridement [11]. While there are no national or international guidelines for the diagnosis of PG, one proposed set requires the fulfillment of 2 major and minor criteria [10]: major criteria (1) rapid progression of a painful ulcer with a violaceous, undermined border and (2) exclusion of other causes of cutaneous ulceration; minor criteria - (1) a history suggestive of pathergy or clinical findings in keeping with cribriform scarring, (2) systemic disease associated with PG, (3) histological findings of neutrophilic dermal infiltrate/mixed inflammation/lymphocytic vasculitis, and (4) a rapid response to systemic corticosteroid therapy.

The patient in our case fulfilled both of the major criteria as well as 3 out of the 4 minor criteria. While there are no treatment guidelines, treatment should encompass local wound care and the inflammatory component. Optimal wound care involves moisture-retentive, occlusive dressings, treatment for secondary infections, and hyperbaric oxygen [12]. Treatments 
to control inflammation include topical agents (corticosteroids [13], tacrolimus [14], and cyclosporine [15]), intralesional agents (corticosteroids [16] and cyclosporine [17]), and systemic agents (oral corticosteroids [18], oral cyclosporine [15], and infliximab [19]). Other biologic agents (etanercept [20] and adalimumab [21]), granulocyte apheresis [22], and thalidomide [23] have been reported to have successful outcomes.

Our case is unique for two reasons. First, the patient did not have any underlying systemic illness typically associated with PG. Second, we are not aware of any previous reports of PG developing at the exit site of a PD catheter - a fact which led to a delay in diagnosis. Fortunately, the insertion of a permcath did not lead to a similar lesion, as pathergy often drives the disease.

\section{Conclusion}

We reported on a case of PG presenting at a PD catheter exit site in an ESRD patient with type 2 diabetes. The diagnosis of PG should be considered with painful, nonhealing lesions unresponsive to antibiotics. A high index of clinical suspicion is needed. Delayed diagnosis leads to worsening of the lesions and has the potential for inappropriate surgical interventions.

\section{Acknowledgements}

The authors acknowledge the Department of Research and Performance Support, RQHR.

\section{Statement of Ethics}

Our institutional REB does not require approval for fewer than 3 cases. Written informed consent was obtained from the patient for publication of this case report and any accompanying images. A copy of the written consent is available for review by the Editor-in-Chief of this journal.

\section{Disclosure Statement}

The authors have no conflicts of interest to declare.

\section{Availability of Data and Materials}

Data sharing is not applicable to this article, as no data sets were generated or analyzed during the current study. 


\section{Author Contributions}

S.A. drafted the manuscript, and K.H. and B.P. edited the manuscript. All authors agreed on the final version of the manuscript.

\section{References}

1 Abdelrahman W, Walsh MY, Hoey SE, O'Kane D. Pyoderma gangrenosum: a rare cause of cutaneous ulceration and one easily misdiagnosed. Case Rep Pediatr. 2016;2016:5971706.

2 Powell FC, Su WP, Perry HO. Pyoderma gangrenosum: classification and management. J Am Acad Dermatol. 1996 Mar;34(3):395-409.

3 La Marca S, Toussoun G, Ho Quoc C, Sebban H, Delay E. Pyoderma gangrenosum: an exceptional complication of venous access device. J Vasc Access. 2014 Jul-Aug;15(4):321-4.

4 Marzano AV, Trevisan V, Lazzari R, Crosti C. Pyoderma gangrenosum: study of 21 patients and proposal of a 'clinicotherapeutic' classification. J Dermatolog Treat. 2011 Oct;22(5):254-60.

5 Miller J, Yentzer BA, Clark A, Jorizzo JL, Feldman SR. Pyoderma gangrenosum: a review and update on new therapies. J Am Acad Dermatol. 2010 Apr;62(4):646-54.

6 Perry HO, Brunsting LA. Pyoderma gangrenosum; a clinical study of nineteen cases. AMA Arch Derm. 1957 Mar;75(3):380-6.

7 Cozzani E, Gasparini G, Parodi A. Pyoderma gangrenosum: a systematic review. G Ital Dermatol Venereol. 2014 Oct;149(5):587-600.

8 Brunsting LA, Goeckerman WH, O'Leary PA. Pyoderma (echthyma) gangrenosum: clinical and experimental observations in five cases occurring in adults. Arch. Derm. Syphilol. 1930;22(4):655-80.

9 Oka M, Berking C, Nesbit M, Satyamoorthy K, Schaider H, Murphy G, et al. Interleukin-8 overexpression is present in pyoderma gangrenosum ulcers and leads to ulcer formation in human skin xenografts. Lab Invest. 2000 Apr;80(4):595-604.

10 Su WP, Davis MD, Weenig RH, Powell FC, Perry HO. Pyoderma gangrenosum: clinicopathologic correlation and proposed diagnostic criteria. Int J Dermatol. 2004 Nov;43(11):790-800.

11 Ahronowitz I, Harp J, Shinkai K. Etiology and management of pyoderma gangrenosum: a comprehensive review. Am J Clin Dermatol. 2012 Jun;13(3):191-211.

12 Chiang IH, Liao YS, Dai NT, Chiao HY, Chou CY, Chen SG, et al. Hyperbaric Oxygen Therapy for the Adjunctive Treatment of Pyoderma Gangrenosum: A Case Report. Ostomy Wound Manage. 2016 May;62(5):32-6.

13 Wenzel J, Gerdsen R, Phillipp-Dormston W, Bieber T, Uerlich M. Topical treatment of pyoderma gangraenosum. Dermatology. 2002;205(3):221-3.

14 Lyon CC, Stapleton M, Smith AJ, Mendelsohn S, Beck MH, Griffiths CE. Topical tacrolimus in the management of peristomal pyoderma gangrenosum. J Dermatolog Treat. 2001 Mar;12(1):13-7.

15 Theissen U, Luger TA, Schwarz T. [Successful topical administration of cyclosporin A in pyoderma gangraenosum]. Hautarzt. 1996 Feb;47(2):132-5.

16 Goldstein F, Krain R, Thornton JJ. Intralesional steroid therapy of pyoderma gangrenosum. J Clin Gastroenterol. 1985 Dec;7(6):499-501.

17 Mrowietz U, Christophers E. Clearing of pyoderma gangrenosum by intralesional cyclosporin A. Br J Dermatol. 1991 Nov;125(5):499.

18 Gettler S, Rothe M, Grin C, Grant-Kels J. Optimal treatment of pyoderma gangrenosum. Am J Clin Dermatol. 2003;4(9):597-608.

19 Brooklyn TN, Dunnill MG, Shetty A, Bowden JJ, Williams JD, Griffiths CE, et al. Infliximab for the treatment of pyoderma gangrenosum: a randomised, double blind, placebo controlled trial. Gut. 2006 Apr;55(4):505-9.

20 Rogge FJ, Pacifico M, Kang N. Treatment of pyoderma gangrenosum with the anti-TNFalpha drug Etanercept. J Plast Reconstr Aesthet Surg. 2008;61(4):431-3.

21 Bardazzi F, Magnano M, Tengattini V, Loi C. Pyoderma gangrenosum in the genital area: successful treatment using adalimumab. Eur J Dermatol. 2018 Apr;28(2):263-4.

22 Russo I, Miotto S, Colpo A, Marson P, Tison T, Ferrazzi A, et al. Successful treatment of pyoderma gangrenosum with granulocyte and monocyte adsorption apheresis. Int Wound J. 2017 Feb;14(1):282-4.

23 Federman GL, Federman DG. Recalcitrant pyoderma gangrenosum treated with thalidomide. Mayo Clin Proc. 2000 Aug;75(8):842-4. 


\section{Case Reports in Nephrology and Dialysis}

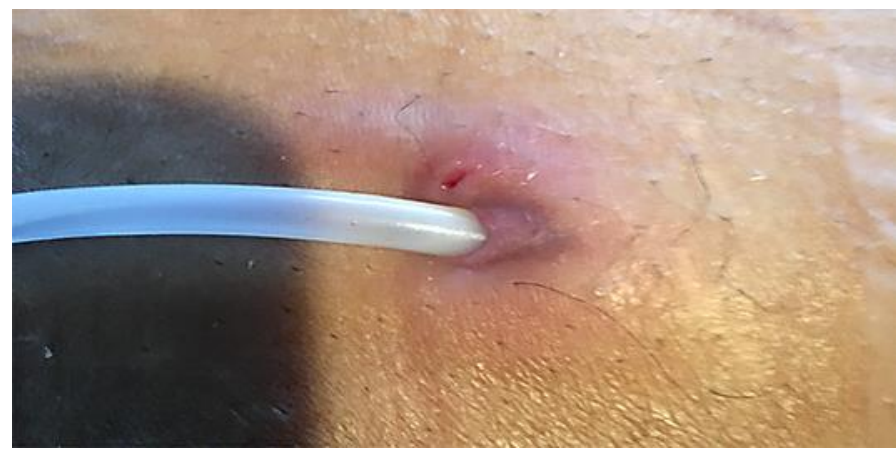

Fig. 1. Initial lesion showing a small ulcerated area surrounded by erythema and mild swelling around the exit site of a peritoneal dialysis catheter.

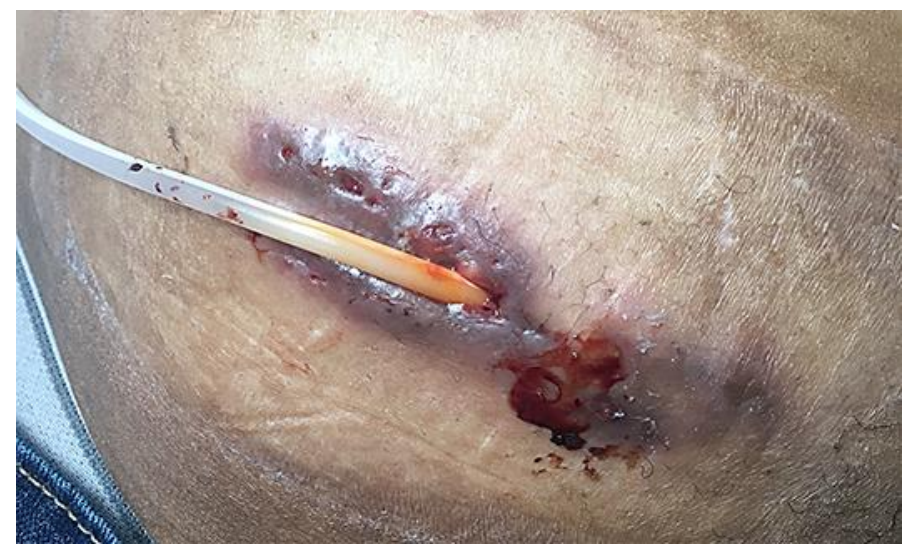

Fig. 2. Four months after the initial lesion had been identified, it had progressed despite topical mupirocin application to the affected skin and intermittent systemic antibiotics.

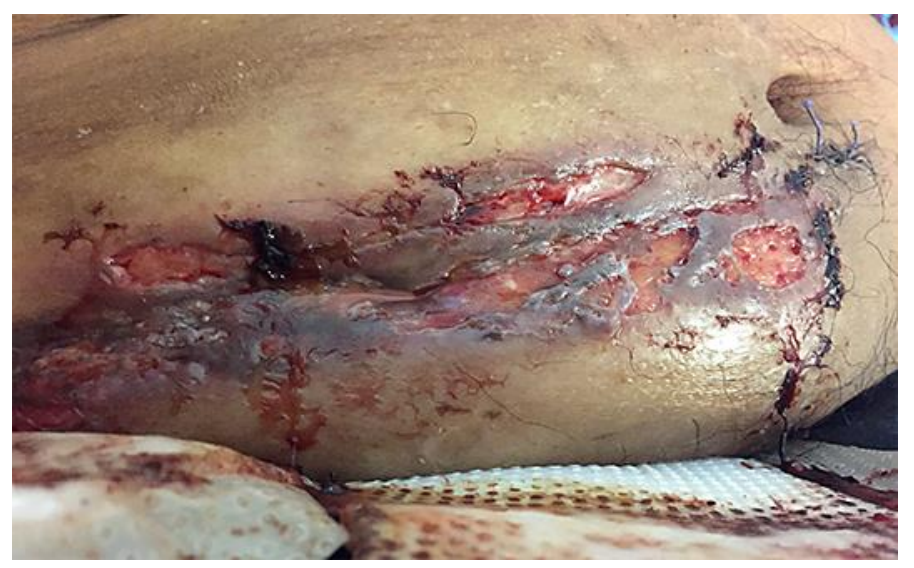

Fig. 3. Twelve months after the initial lesion had been identified, there was blistering of the epidermis overlying the lesion, resulting in a violaceous, ulcerated appearance with visible subcutaneous tissue below at the time of PD catheter removal. 


\section{Case Reports in Nephrology and Dialysis}

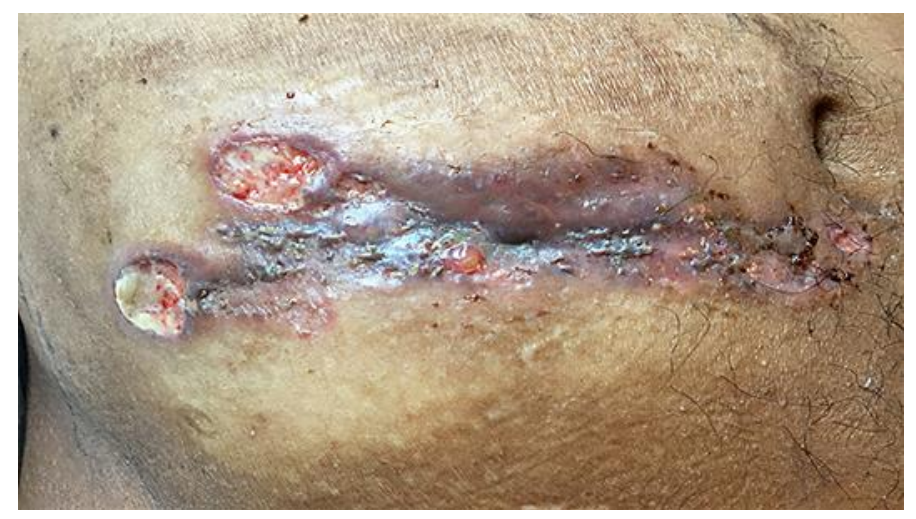

Fig. 4. Two weeks after treatment with topical clobetasol ointment.

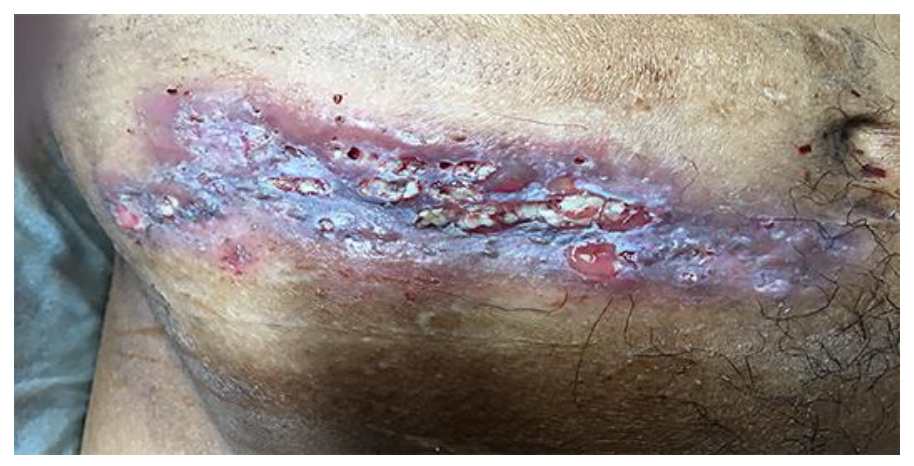

Fig. 5. After 2 months of topical and systemic steroids.

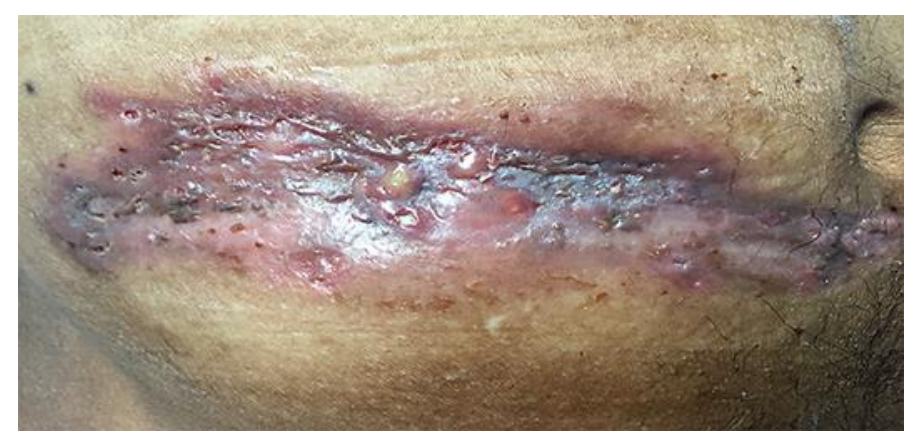

Fig. 6. Two months after oral cyclosporine treatment. www.karger.com/cnd 